\title{
Local and primary controls of a multi-terminal HVDC grid in an experimental setup.
}

\author{
M. Jiménez Carrizosa ${ }^{1}$, A. Arzandé ${ }^{2}$, A. Benchaib ${ }^{3}$, G. Damm ${ }^{4}$, E. Berne $^{5}$, \\ P. Egrot $^{5}$, JC. Vannier ${ }^{2}$ and F. Lamnabhi-Lagarrigue ${ }^{1}$ \\ ${ }^{1}$ LSS CentraleSupélec, France, email: Miguel.Jimenez@1ss.supelec.fr, lamnabhi@1ss.supelec.fr \\ ${ }^{2}$ CentraleSupélec, France, email: Amir.Arzande@centralesupelec.fr, \\ ${ }^{3}$ ALSTOM GRID / Cnam, France, email: abdelkrim.benchaib@alstom.com \\ ${ }^{4}$ IBISC, France, email: gilney.damm@ibisc.fr \\ ${ }^{5}$ EDF, France, email: erik.berne@edf.fr, philippe.egrot@edf.fr
}

\begin{abstract}
Keywords
$<<$ MT-HVDC $>>,<<$ VSC $>>,<<$ Droop control $>>$
\end{abstract}

\begin{abstract}
This paper focuses on the experimental implementation of local and primary controls for MTHVDC in a real test bench $\left(P_{\text {nom }}=6 \mathrm{~kW}\right)$. A local control with two different time scales, one for the current and the other for the voltage, and primary control (droop control), which is responsible to vary the reference voltage when a disturbance appears in the network, are addressed in this paper.
\end{abstract}

\section{Introduction}

In our days $\mathrm{AC}$ grids are reaching their limits mainly because power consumption has greatly increased. Besides, the gradual integration of renewable energies, with variable nature, makes the network less stable. Parallel to these events, the number of blackouts has been intensified [1]. It is under this framework a where the researcher are seeking alternative solutions for the energy transport and distribution, where HVDC grids could play a key role in the future [2].

DC networks have some advantages over AC grids, such as the absence of reactive power, and therefore lower losses in power transmission, or the absence of skin effect in the cables, to name a few [2]. DC networks can also be used to join AC networks with different frequencies. Besides, the experience has revealed that blackouts are not propagated from one AC side to the other through these DC bypass lines [3]. However, the majority of operating DC grids only connect two nodes, in back to back mode operation, and the study of MT-HVDC networks is still an open field, mainly because it has not been developed and implemented with efficacy a DC-circuit breaker $[4,5]$. Parallel to this problem, the efforts are also devoting to study how to implement a whole control in order to make operational MT-HVDC networks as soon as the technology will be ready. The control philosophy which is imposing includes local, primary, secondary and tertiary controllers, in resemblance to classic hierarchical AC control [6], as it is shown in table I.

This paper focuses on the experimental implementation of local and primary controls in a MT-HVDC grid. It will show as, thanks to the applied control techniques, it is possible to alleviate the consequences when a power perturbation appears without need of communication between the nodes.

This work is outlined as follows: Section II presents the local control explanation. In Section III the primary control is exhibited. In Section IV the test bed is explained. In section Section $\mathbf{V}$ the results are shown. Finally, in Section VI the conclusions are explained. 
Table I: Philosophy control.

\begin{tabular}{|c|c|}
\hline AC & DC \\
\hline Tertiary & Tertiary \\
$(+15$ min $)$ & $(+15$ min $)$ \\
\hline Secondary & Secondary \\
$(\sim$ min $)$ & $(\sim$ min $)$ \\
\hline Primary & Primary \\
$(\sim$ ms $)$ & $(\sim$ s $)$ \\
\hline Local & Local \\
& $(\sim$ ms $)$ \\
\hline
\end{tabular}

\section{Local control of a MT-HVDC system}

The local controller is responsible to control the VSC converters, see [7, 8]. It operates in a time range of milliseconds. Two possible control philosophies could be carried out depending on node operation. There will be VSC nodes where the DC voltage and reactive AC power will be controlled, and other nodes where the local controller is responsible to regulate the active and reactive power.

If we desire to control the pair DC voltage and reactive power, the local controller is composed by the so-called inner and outer controllers [9]. The first one is responsible to control the currents in the VSC, which have a dynamic much faster than the DC voltage, which is regulated by the outer controller. This second controller gives the references to the inner controller. On the contrary, if we want to handle the active and reactive power, the local controller of VSC is only composed by the inner controller.

\subsection{Inner controller}

The inner controller is responsible to control the active and reactive power thanks to the control of the current. If the control is implemented in the $d q$ frame, we will control the active power controlling the current $i_{d}$, and we will control the reactive power controlling $i_{q}$. The simplest control loop consists of two PI controllers, respectively for $d$ and $q$ axis current control, as figure 1 shows.

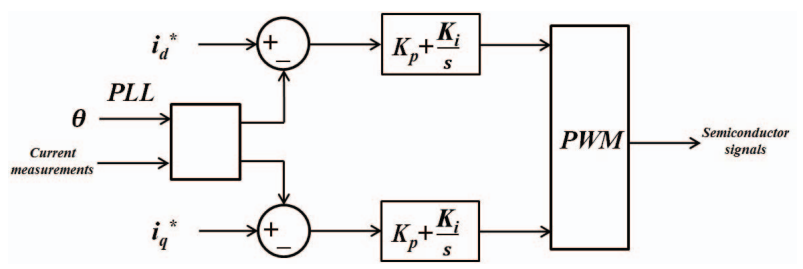

Figure 1: Inner controller.

In order to apply the $d q$ transformation, it is necessary to know the value of the angle of the AC voltage for all moments. This value is computed by using the well-known phase lock loop (PLL) synchronization technique. The corresponding signals to the switches are generated by means of Pulse Width Modulation (PWM) technique [10].

\subsection{Outer controller}

The DC voltage has a dynamic much slower than the current. The control consists of one PI controller, as figure 2 shows. This controller is responsible for the DC voltage tracks its reference given by the primary controller.

\section{Primary Controller}

The primary control operates in a time range of seconds. In DC systems, there are some suitable candidates for primary control [11], we have chosen droop control to carry out this task [12]. Droop control is distributed, and it employs the droop mechanism to regulate the DC voltage which adapts the power 


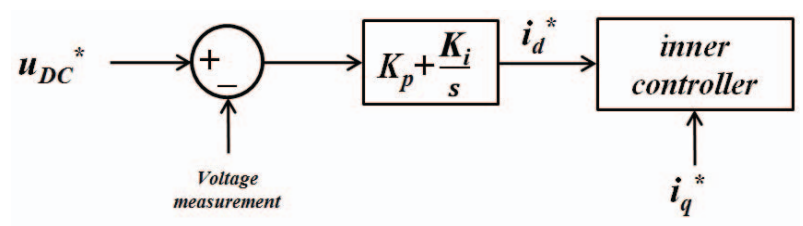

Figure 2: Outer controller

injections in each node where the controller is implemented (droop control usually is not implemented in all nodes). It is a robust distributed controller for MT-HVDC systems without needing a communication system, which permits to operate each one autonomously.

Droop control reacts to an imbalance and it can be explained as follows. When there is a variation in the grid, in the node where the controller exits, they adjust the amount of power and voltage level via droop control, see figure 3. This control is a set of local proportional controllers with gain $k$, called droop gain. This local droop gain is also the slope of the line in figure 3. The value of droop gain depends on the size of each node's reserve, and it gives an idea of how much power provides each node when a variation occurs, in function of its capabilities. From an automatic control point of view, it represents a state feedback only using local measurements [13]. Each $k$ will be designed in a global way, such as to attain stability of the whole system, but will act in a local way.

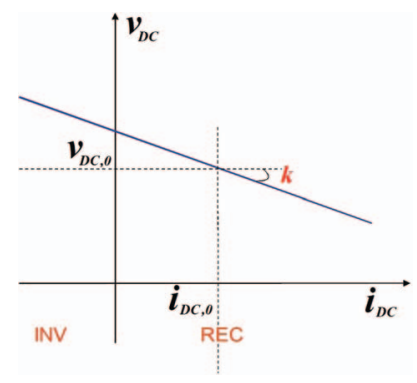

Figure 3: Droop control.

Each droop controller for the converter $j$ is simply expressed as:

$$
i_{D C, j}^{*}=i_{D C, j}+k_{j} \cdot\left(u_{D C, j}-u_{D C, j}^{*}\right) \forall j \in \Delta .
$$

where $i_{D C, j}$ is the DC output current, $k_{j}$ is the droop gain with dimensions of $\left[\Omega^{-1}\right]$ and $u_{D C, j}$ is the DC voltage in the capacitor of the DC side for the converter $j$. Finally $\Delta$ is the set of nodes where droop control is implemented.

The primary control is usually implemented in nodes where energy reserve exists. In this case, the only possible philosophy for the local control will be to control the pair DC voltage/ reactive power. The droop control will give the DC voltage reference for the local controller.

The dynamic interaction between both controllers is usually neglected, because the internal dynamic of the converters are much faster than the droop control.

\subsection{Multi-Terminal HVDC grid}

Before to study in depth the droop control, we must define the state variables involved in the model. According to [12], a MT-HVDC network can be represented as the interconnection of nodes and branches. The nodes come in three different forms: injection nodes (or power-input nodes), consumption nodes (or power-output nodes) and interconnection nodes.

From the point of view of primary, we can neglected the internal dynamics of the converters, consequently the external grids (AC or DC) are modelled as current sources. Furthermore, the internal branches are modelled by $\pi$-equivalent circuits. Therefore, a general configuration of a MT-HVDC is shown in figure 4. 


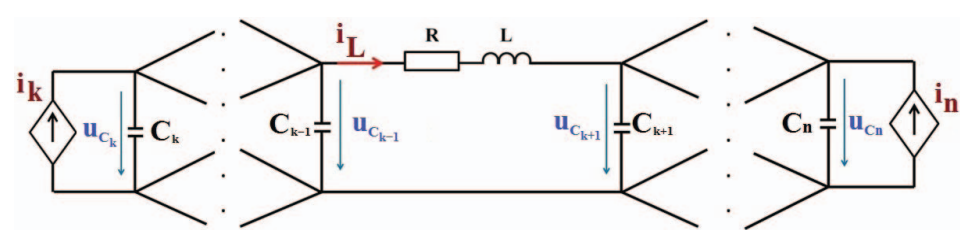

Figure 4: General configuration of a MT-HVDC network.

With this configuration adopted, the behaviour of any MT- HVDC grid can be described only in terms of capacitors, inductances, resistances and current sources. Thus it is possible to find a linear state-space representation, where the state variables are the voltages in the capacitors and the currents through the inductances, and the inputs are the currents on the sources.

As we have already mentioned, droop control is not applied in all nodes, it is usually implemented in nodes which have spare energy (usually called primary reserve). For example, if an external grid is a large AC network, then it can inject or absorb power with less restrictions, and it is more natural to implement the droop there. Even more if other nodes are connected to grids with high variability, as for example renewable energy nodes.

After all the aforesaid, the inputs could be divided in two sets. In the first set $u$ are the variables that can be used to control the system (nodes where there is droop control), and in the other set, $w$, there are the variables that are not possible to manipulate (nodes where there is no droop control). These last variables could also be treated as external disturbances. In the same way we split the output in two sets. One set contains the variables that can be used in the control $(v)$, and the other contains the variables that are not employed by the controller $(y)$.

If the system has $i$ injection nodes, $j$ consumption nodes, $p$ interconnection nodes and $l$ branches, the state-space is defined as:

$$
\left\{\begin{aligned}
\dot{x} & =A x+B_{u} u+B_{w} w \\
v & =C_{v} x \\
y & =C_{y} x
\end{aligned}\right.
$$

with $x=\left[u_{C_{1}}, \cdots, u_{C_{i+j+p}}, i_{L_{1}}, \cdots, i_{L_{l}}\right]^{T}, A \in \mathbb{R}^{(i+j+p+l) \times(i+j+p+l)}, B_{u} \in \mathbb{R}^{(i+j+p+l) \times i}, B_{w} \in \mathbb{R}^{(i+j+p+l) \times j}$, $C_{v} \in \mathbb{R}^{i \times(i+j+p+l)}$, and $C_{y} \in \mathbb{R}^{j \times(i+j+p+l)}$. Where $u_{C 1}, \cdots, u_{C_{i+j+p}}$ are the voltages in the capacitors, and $i_{L_{1}}, \cdots, i_{L_{l}}$ are the currents through the inductances.

It should be noted that the size of matrix $B_{u}, B_{w}, C_{v}$ and $C_{y}$ could be changed if the droop controllers are applied in other nodes than injection nodes. But the most common case is the proposed one.

\subsection{Calculation of $\mathrm{K}$ with LQR technique.}

As have been explained in [13], $k_{j}$ must be negative in order to guarantee the stability (asymptotically) of the system. However, it says nothing about the size of $k_{i}$, the droop gain of node $i$. These gain values can be obtained with LQR methods, because as the droop control can be seen as a state feedback limited to local measurements. They can be optimized by means of optimal control techniques as linear-quadratic regulator (LQR) [14]. LQR strategy has as main advantages its robustness and the possibility to weight different variables of the control inputs and the state vector. This means that the controller will place less or more emphasis on stabilizing a desired variable following physical considerations of the system. This algorithm optimizes the control with respect to a cost function, $J$. This function is quadratic, and it is often defined as a sum of the deviations of key measurements from their desired values, and assures a minimum energy consumption to reach the equilibrium point.

Considering the variation respect to equilibrium point in system (2) $\left(x=x^{*}+\tilde{x}\right.$ and $\left.u=u^{*}+\tilde{u}\right)$, we obtain:

$$
\dot{\tilde{x}}=A \tilde{x}+B_{u} \tilde{u}
$$


For an infinite-horizon continuous-time linear system its expression is given by (4).

$$
J=\int_{0}^{\infty}\left(\tilde{x}^{T} Q \tilde{x}+\tilde{u}^{T} \mathcal{R} \tilde{u}\right) d t
$$

where $Q$ is, at least, positive-semidefinite and $\mathcal{R}$ is positive definite.

Therefore, the feedback control that minimizes the value of the cost, function (4), is $\tilde{u}=-F \tilde{x}$, where $F$ is given by equation (5), and $P$ is found by solving the continuous time algebraic Riccati equation $A^{T} P+P A-P B_{u} \mathcal{R}^{-1} B_{u}^{T} P+Q=0$.

$$
F=\mathcal{R}^{-1} B_{u}^{T} P
$$

Also it holds that $\lim _{t \rightarrow \infty} \tilde{x}(t)=0$ and $\lim _{t \rightarrow \infty} \tilde{u}(t)=0$, and since,

$$
\left.\begin{array}{c}
v=C_{v} x \\
v_{r e f}=C_{v} x^{*}
\end{array}\right\} \Rightarrow v-v_{r e f}=C_{v}\left(x-x^{*}\right)=C_{v} \tilde{x}
$$

Taking $u=K\left(v-v^{*}\right)$, then:

$$
u=K C_{V} \tilde{x}
$$

and therefore $\lim _{t \rightarrow \infty} u(t)=0$, so $u^{*}=0$, and consequently $u=\tilde{u}$. Afterwards, we obtain:

$$
u=-F \tilde{x}
$$

and as this two conditions (7) and (8) hold $\forall \tilde{x}$, then $F=-K C_{v}$, and from equation (5) the droop matrix $K$ holds that:

$$
K C_{v}=-\mathcal{R}^{-1} B_{u}^{T} P
$$

Equation (9) shows that, if matrix $K$ is diagonal, then the droop value at each node depends on the value of the node capacitor, this is consistent with the idea expressed in [15], which says that the real gain must be consistent with the value of the capacitor of the DC side of the converter.

\section{Test bench explanation}

The experiments have taken place in the facilities of the Department of Power \& Energy Systems of CentraleSupélec. The test bed has been constructed based on an original setup from EDF. It has 4 terminals as figure 5 shows, which can work at a maximum of $400 \mathrm{~V} \mathrm{DC}$, and with a maximum allowed current of $15 \mathrm{~A}$. The HVDC lines are simulated by variable resistors, therefore we have the ability to vary the length of the represented lines. Each terminal node is connected to an AC grid of $127 \mathrm{~V}$ (rms phase to phase) and $50 \mathrm{~Hz}$. Afterwards, the AC voltage is decreased to $50 \mathrm{~V}$ (rms phase to phase) by means to $\Delta / d$ step-down transformer (see table II for parameters).

\subsection{VSC}

The VSC convertes carry out the AC/DC transformation. The converters are able to operate in both current senses, and they are controlled by means of dSPACE ${ }^{\circledR}$ software package, by the interface of MATLAB SIMULINK ${ }^{\circledR}$ files in real time, thanks to the ControlDesk ${ }^{\circledR}$ software.

In each terminal node of the grid there is a VSC, which is a two-level, three phases converter as figure 6 a shows. In each leg there is a commercial IGBT module (see figure $6 \mathrm{~b}$ ). The module is made of 2 IGBTs with an anti-parallel diode, connected in series, of which the middle point is at terminal 1 (in figure 6b). The IGBT used here is a commercial device: SEMITEACH-IGBT, SKM 50 GB 123D-SKD 51-P3/250F [16].

In figure 11 the real configuration of a VSC converter is shown in the test bench, where we can observe the capacitors, the IGBTs, the inductances (in the AC side) and the current sensors. 

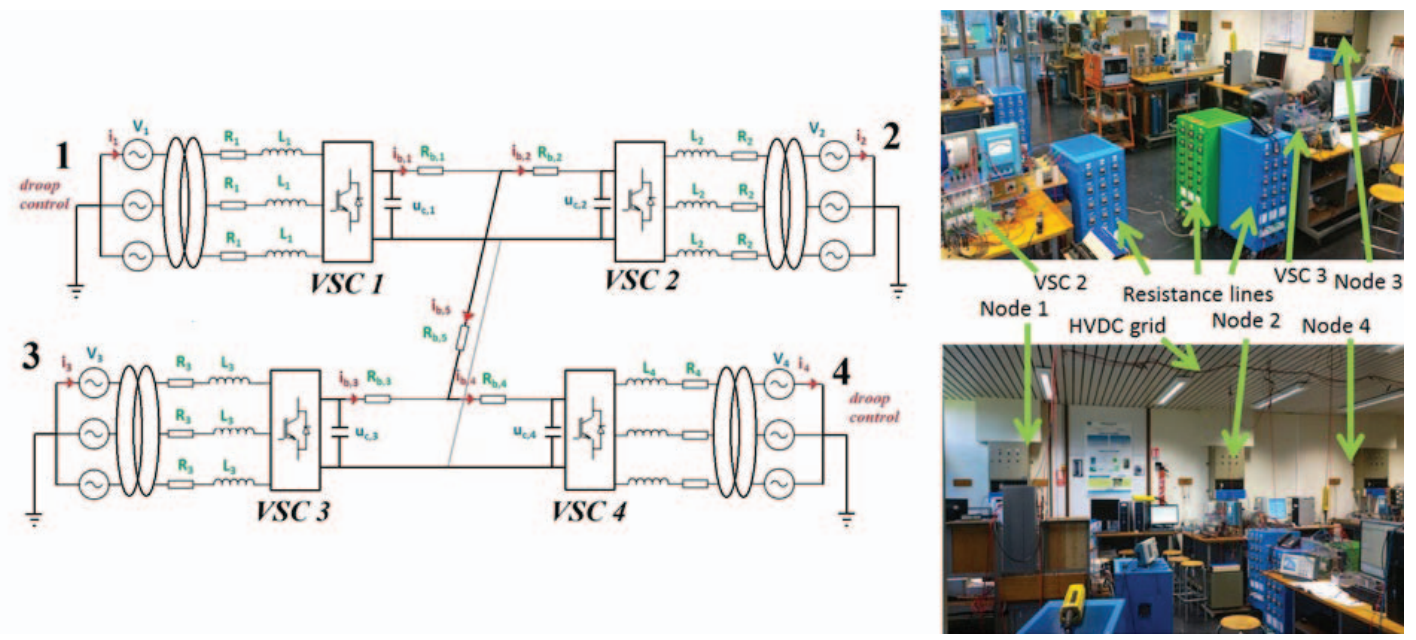

Figure 5: Test bench network. Electrical scheme.
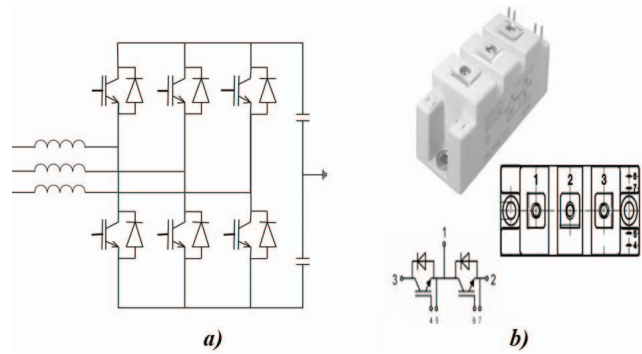

Figure 6: a) VSC scheme

b) IGBT module. Source [16].

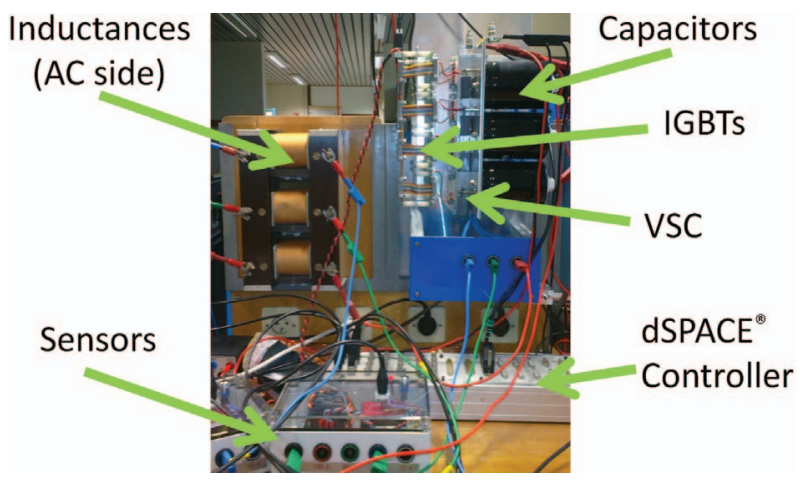

Figure 7: VSC. Real converter.

\subsection{Transformer.}

A $\Delta / d$ step-down transformer, see figure 8 , is used to reduce the $\mathrm{AC}$ voltage in order to achieve the ideal value for the proper operation point of the VSC converters. Besides, it is employed to provide electric galvanic isolation to the system. Its parameters, shown in table II, were obtained after the short-circuit and open-circuit tests.

\subsection{Current Sensor.}

The current sensors have been produced manually in CentraleSupélec. Their characteristics are shown in table III (see figure 9a). The current transducer is the commercial LT 100-S/SP30 from LEM ${ }^{\circledR}$ company (see figure 9b) [17]. 


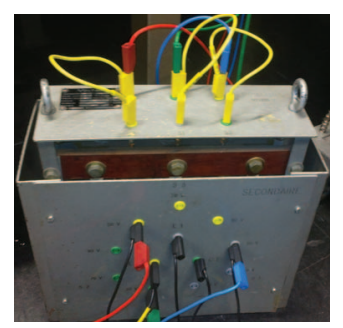

Figure 8: Transformer $\Delta / d(127 / 50 \mathrm{~V})$.

Table II: Transformer parameters.

\begin{tabular}{|c|c|c|}
\hline \multicolumn{3}{|c|}{ Transformer $\Delta / d$} \\
\hline Transformer ratio & $n$ & $127 / 50$ \\
\hline Primary voltage & $V_{p}$ & 127 \\
\hline Secondary voltage & $V_{p}$ & 50 \\
\hline Apparent nominal power & $S_{n}$ & $3500 \mathrm{VA}$ \\
\hline Foucault Losses & $R_{f}$ & $222.1 \Omega$ \\
\hline Magnetizing inductance & $L \omega$ & $41.4 \Omega$ \\
\hline Short-circuit voltage & $U_{s c}$ & $5.7 \mathrm{~V}\left(4 \% U_{n}\right)$ \\
\hline
\end{tabular}

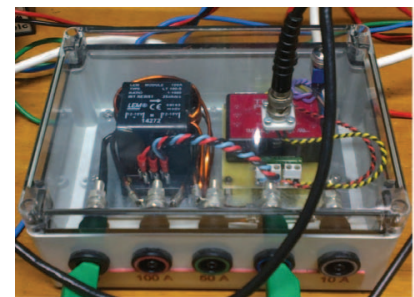

a)

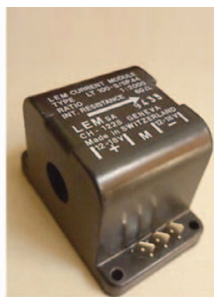

b)

Figure 9: a) Current Sensor. b) Current Transducer LT 100-S/SP30. LEM ${ }^{\circledR}$.

Table III: Current Transducer LT 100-S/SP30. LEM ${ }^{\circledR}$.

\begin{tabular}{|c|c|}
\hline Ouput Type & Instantaneous \\
\hline Technology & Closed loop Hall Effect \\
\hline Measurement & Current \\
\hline Primary Nominal Value & $100 \mathrm{~A}$ \\
\hline Accuracy & $0.5 \%$ \\
\hline Measuring Range & $200 \mathrm{~A}$ \\
\hline Supply Voltage & $12-18$ \\
\hline Secondary Signal & $100 \mathrm{~mA}$ \\
\hline
\end{tabular}

\section{4 dSPACE software and hardware.}

We have used DS1104 R\&D controller card (figure 10) in order to implement the real-time control on the VSC converters, where analogue signals from sensors are converted in digital signals for their processing in the PC, and after signal processing on the computer, these digital signals are sent to the controller card for its conversion in analogue signals with the purpose of implementing the PWM. This card makes our PC a powerful development system for rapid prototyping of control laws. The Real-Time Interface includes SIMULINK ${ }^{\circledR}$ blocks for graphic configuration of input and output by means of ControlDesk ${ }^{\circledR}$ software. 


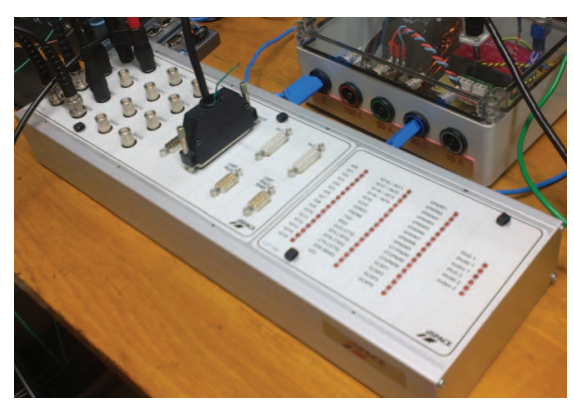

Figure 10: dSPACE 1104 controller card.

\section{Results}

In this section the experimental results are shown. Nodes 1 and 4 are responsible to maintain the voltage level of the network by means of droop control philosophy, nodes 2 and 3 are responsible to maintain the injection/absorption of power thanks to the current control. In table IV all the parameters for the four nodes are summarized, where the node numeration is associated with figure 5 .

Table IV: Test bench parameters.

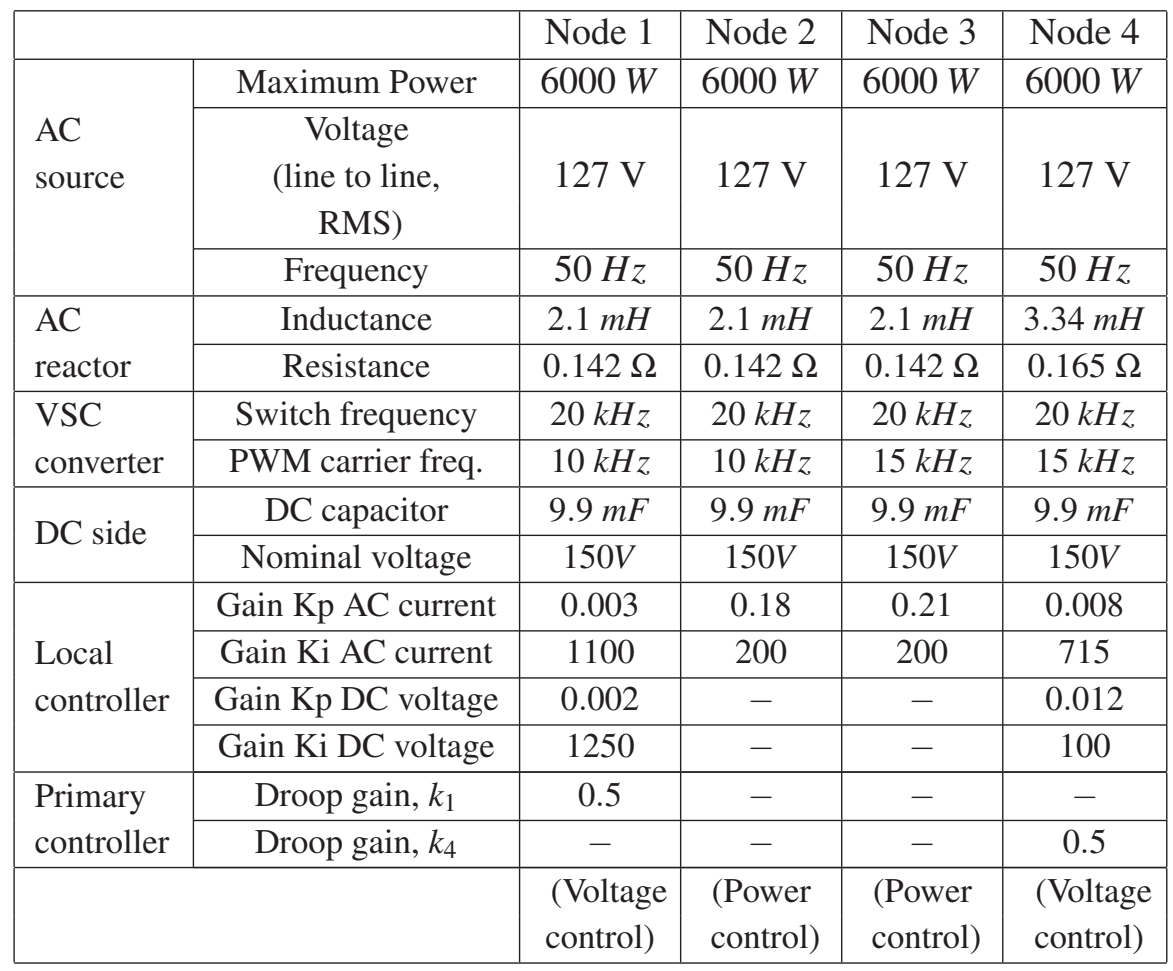



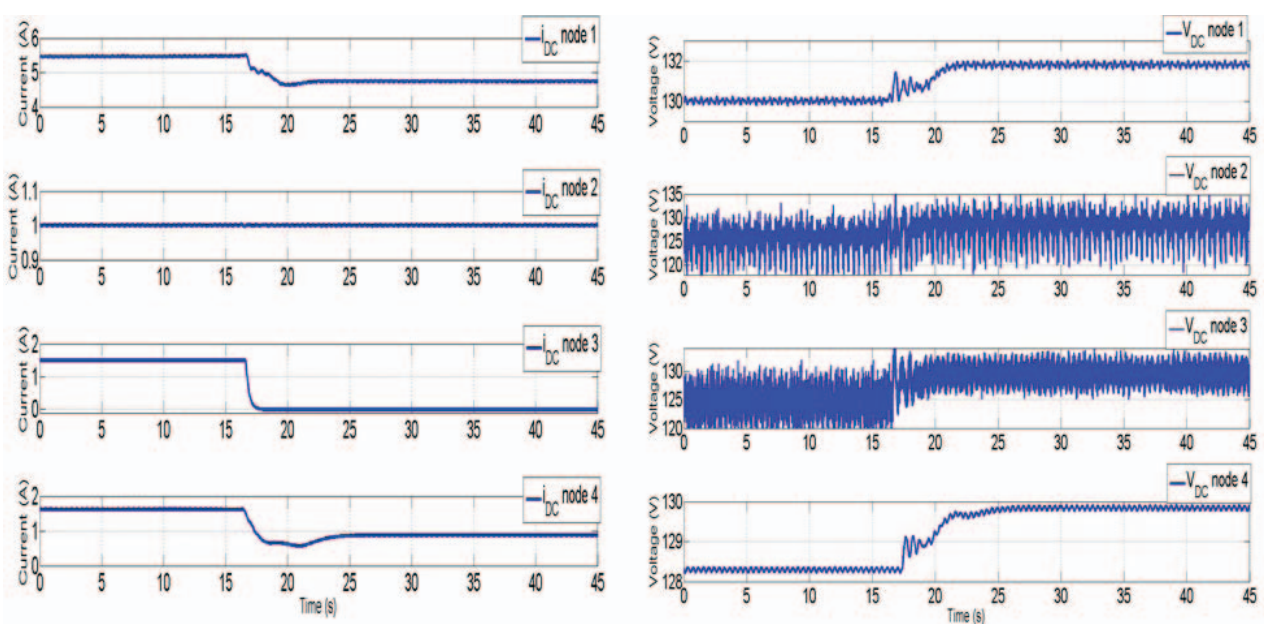

Figure 11: a) DC currents b) DC voltages .

In figure 11 the results are shown. Starting from an equilibrium state in $t=0$, in $t=16$ the power generation falls down suddenly at node 3 , and consequently the current falls down in this node too (from $1.5 \mathrm{~A}$ to $0 \mathrm{~A}$ ). Consequently the sensors of the other nodes sense a variation in the current due to disturbance. In this instant, the droop control acts at the nodes where it is implanted (1 and 4) and internally it adjusts its voltage level following the law established by the droop gain $k_{i}$ until equilibrium is reached around $(t=22 s)$.

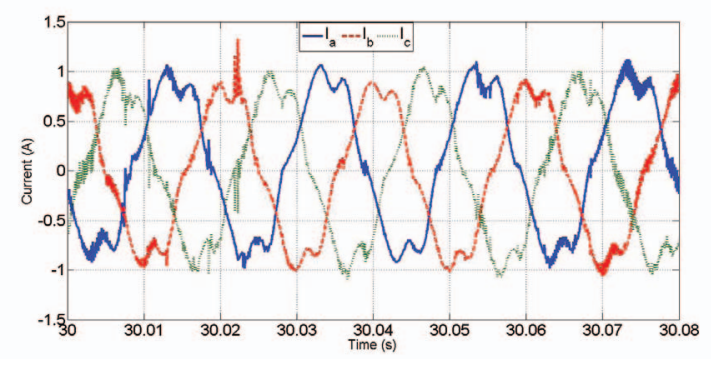

Figure 12: AC currents node 4.

In figure 12, the $\mathrm{AC}$ currents at node 4 are shown. We can appreciate how the signals are almost sinusoidal.

\section{Conclusions}

In this paper the experimental implementation of local controllers (VSC converters) and primary control (droop control) for a four terminal HVDC test bench is shown. Thanks to this philosophy of control, the system is able to respond satisfactorily to network disturbances without communication between nodes, and only with local measurements.

\section{References}

[1] G. Beck, D. Povh, D. Retzmann, and E. Teltsch, "Global blackouts- lessons learned," in Siemens review, July 2011.

[2] J. Arrillaga, Y. Liu, and N. Watson, Flexible Power Transmission. The HVDC Options. John Wiley \& Sons Ltd, 2007. 
[3] O. Mousavi, L. Bizumic, and R. Cherkaoui, "Assessment of hvdc grid segmentation for reducing the risk of cascading outages and blackouts," in Bulk Power System Dynamics and Control - IX Optimization, Security and Control of the Emerging Power Grid (IREP), 2013 IREP Symposium, 2013.

[4] D. Jovcic, D. Van Hertem, K. Linden, J.-P. Taisne, and W. Grieshaber, "Feasibility of DC transmission networks," in Innovative Smart Grid Technologies (ISGT Europe), 2011 2nd IEEE PES International Conference and Exhibition on, Dec 2011, pp. 1-8.

[5] M. Jiménez Carrizosa, J. Cortés, A. Benchaib, P. Alou, G. Damm, J. A. Cobos, and F. LamnabhiLagarrigue, "DC/DC converters as DC circuit-breakers in HVDC networks operation." in Power Electronics and Applications (EPE), 2014 16th European Conference on, August 2014, pp. 1-10.

[6] P. Kundur, Power systems stability and control, 1st ed. Mc Graw Hill, 1993.

[7] J.-L.Thomas, S. Poullain, and A.Benchaib, "Analysis of a robust DC-bus voltage control system for a VSC transmission scheme," in AC-DC Power Transmission, 2001. Seventh International Conference on (Conf. Publ. No. 485), Nov 2001, pp. 119-124.

[8] Y. Chen, J. Dai, G. Damm, and F. Lamnabhi-Lagarrigue, "Nonlinear control design for a multiterminal VSC-HVDC system," European Control Conference (ECC), 2013, pp. 3536-3541, July 2013.

[9] C. Bajracharya, "Control of VSC-HVDC for wind power," Norwegian University of Science and Technology, June 2008.

[10] D. G. Holmes and T. A. Lipo, "Pulse width modulation for power converters: principles and practice," John Wiley \& Sons, 2003.

[11] R. Hassan, S. Shah, and J. Sun, "Review of control methods for HVDC transmission systems," 2013.

[12] E. Prieto-Araujo, F. B. A.Junyent-Ferré, and O. Gomis-Bellmunt, "Methodology for droop control dynamic analysis of multi-terminal vsc-hvdc grids for offshore wind farms," IEEE Transactions on power delirey, vol. 26, no. 4, pp. 2479-2485, October 2011.

[13] M. Jiménez Carrizosa, G. Damm, A. Benchaib, and F. Lamnabhi-Lagarrigue, "Distributed primary droop control in multi terminal high voltage direct current networks," IEEE ISIE 2015, the 24th IEEE International Symposium on Industrial Electronics, Rio de Janeiro, Brazil June 3-5, 2015. .

[14] B. Anderson and J. Moore, "Optimal control- linear quadraticc methods," Prentice Hall, 1989.

[15] P. Rault, "Dynamic modeling and control of multi-terminal HVDC grids," Ph.D. dissertation, Ecole Centrale de Lille, 2014.

[16] SEMIKRON, "IGBT power electronics teaching system principle for sizing power converters," www.semikron.com, 2008.

[17] LEM, "Current transducers," http://www.lem.com, 2008. 